Revue

d'ethnoécologie
Revue d'ethnoécologie

$17 \mid 2020$

Varia

\title{
Arbiț ou la purée d'herbes sauvages des Babors
}

Une étude ethnolinguistique d'une recette menacée

Arbit the wild grass mash of the Babor Mountains: an ethnolinguistic survey of a threatened recipe

\section{Massinissa Garaoun}

\section{OpenEdition}

\section{Journals}

\section{Édition électronique}

URL : https://journals.openedition.org/ethnoecologie/5882

DOI : $10.4000 /$ ethnoecologie.5882

ISSN : 2267-2419

\section{Éditeur}

Laboratoire Eco-anthropologie et Ethnobiologie

\section{Référence électronique}

Massinissa Garaoun, «Arbiț ou la purée d'herbes sauvages des Babors », Revue d'ethnoécologie [En ligne], 17 | 2020, mis en ligne le 30 juin 2020, consulté le 26 mai 2021. URL : http:// journals.openedition.org/ethnoecologie/5882 ; DOI : https://doi.org/10.4000/ethnoecologie.5882

Ce document a été généré automatiquement le 26 mai 2021.

\section{(†)

Revue d'ethnoécologie est mis à disposition selon les termes de la licence Creative Commons Attribution - Pas d'Utilisation Commerciale - Pas de Modification 4.0 International. 


\title{
Arbit ou la purée d'herbes sauvages des Babors
}

\author{
Une étude ethnolinguistique d'une recette menacée \\ Arbit the wild grass mash of the Babor Mountains: an ethnolinguistic survey of \\ a threatened recipe
}

Massinissa Garaoun

\section{Introduction}

1 Une purée d'herbes sauvages cueillies à travers collines et vallons, avant d'être nettoyées à l'eau de source, hachées à la faucille, jetées dans de l'eau bouillante salée, puis servies avec de l'huile d'olive ou du beurre : voilà l'objet qui nous intéressera ici. Un plat offert à qui se donnera la peine de partir en quête des nombreux simples qui le composent, en prenant soin d'identifier les espèces comestibles et d'en sélectionner les meilleures parties. Autrefois synonyme de période de disette, la consommation de arbit s'est figée dans le statut de plat régional traditionnel des Babors; et en particulier dans sa capitale régionale, Jijel, au point d'être aujourd'hui considérée par les Jijéliens comme une authentique recette du pays des Babors.

2 Ce plat sans doute très ancien, de facture simple bien que nécessitant une certaine connaissance de la flore sauvage, est intéressant à plus d'un titre. Sur le plan de l'étude de l'environnement, il permet de renouer, le temps d'une cueillette, avec son environnement naturel direct, un modèle de nutrition entièrement écologique. Sur le plan ethnobiologique, arbit est un véritable symbole du rapport symbiotique des habitants d'un massif montagneux et de leur savoir accumulé sur les milieux qui les entourent. Du point de vue culinaire, notre purée est, selon les nombreuses combinaisons d'herbes possibles, l'occasion de générer et de découvrir la gamme de saveurs que peuvent offrir des plantes sauvages; et il serait sans doute intéressant de regarder les valeurs nutritionnelles de ce type d'alimentation. Enfin, l'aspect linguistique qui nous intéressera tout particulièrement ici, est également très 
important: la description des différentes recettes de arbit pourrait être en effet l'occasion de la réalisation d'un véritable atlas phytonomique de la région, la réalisation de cette recette requérant la mobilisation d'une certaine connaissance de la botanique locale. Nos résultats nous permettront de croiser les questions de la linguistique du contact, s'intéressant ici à la rencontre et l'interpénétration linguistique millénaire ${ }^{1}$ des deux langues pratiquées dans le massif des Babors - l'arabe et le berbère -, et celles de l'ethnolinguistique, cherchant à comprendre la motivation des formes sémantiques observées au moyen du prisme ethnologique.

Après avoir présenté la recette de arbiț, ses variantes et des recettes proches attestées en Afrique du Nord, nous présenterons ses différentes dénominations à travers le massif des Babors. Nous regarderons ensuite les différentes herbes entrant dans la composition de arbit et tenterons de retracer les origines étymologiques des noms de celles-ci dans les deux langues de la région, l'arabe préhilalien du massif des Babors (dit žǐ̌lìya, arabe jijélien [AJ]), et le berbère de la même région (dit țasahlit [TS] ou kabyle extrême-oriental). Enfin, nous questionnerons l'avenir et les techniques de valorisation qui pourraient être mises en place pour assurer la prospérité de ce plat.

Nous tenons à avertir le lecteur potentiellement attiré par la confection de arbiṭ du réel danger pour le non-connaisseur de la flore sauvage, d'intoxication alimentaire par des végétaux entièrement ou partiellement non comestibles. Cette recette requérant un savoir traditionnel héritée d'une culture experte de son environnement naturel, nous invitons les potentiels amateurs à prendre contact avec des héritiers de ces savoirs, plutôt que de tenter par eux-mêmes de récolter et de cuisiner sans précautions des végétaux sauvages potentiellement toxiques.

\section{Méthodologie}

$5 \quad$ Notre étude se base sur une enquête par entretiens semi-directifs auprès de locuteurs arabophones et berbérophones natifs du pays des Babors. Dans un premier temps, nous avons questionné au total une quinzaine de locuteurs sur l'existence ou non d'une purée à base d'herbes sauvages dans leur localités respectives et, si oui, sur sa composition. La plupart des réponses ont été positives, ce qui nous a amené dans un second temps à demander des précisions sur les recettes précises de chaque localité, les plantes utilisées et leurs dénominations, ainsi que l'extension éventuelle de cette purée dans la culture locale (littérature orale, etc.). L'identification des espèces végétales utilisées dans cette recette s'est faite au moyen de photographies de celles-ci (inflorescences et feuillage) réalisées dans la région en 2017.

6 Les localités (confédération + commune) dans desquelles nous avons interrogé des natifs sont d'ouest en est : Aït Melloul (Tichy), Aït Smaïl (Bordj Mira), Aït Mella (Oued El Bared), Aït Bouyoucef (Tamridjet), Amouchas, Ait Segoual (Ziama-Mansouriah), Bni Mâad (Ziama-Mansouriah), Bni Aïssa (Ziama-Mansouriah), Jijel-ville, Oulad Taffer (Jijel), Bni Hbibi. Nous avons pu récolter les recettes dans huit de ces douze localités : quatre localités berbérophones et quatre arabophones. Nous souhaitons par ailleurs remercier tout particulièrement quatre informateurs qui nous ont grandement aidé dans la réunion de ce corpus: Hamza Azni, Hocine Slimoune, Mehdi Mimoune et Nadia Garaoun.

7 Nous avons procédé à l'analyse du corpus de recettes ainsi récoltées, en les comparant entre elles et avec des plats ressemblants attestés dans les massifs voisins du Djurdjura, 
de Collo et de l'Atlas blidéen. Enfin, nous avons mobilisé les quelques ressources numériques concernant cette recette, des vidéos présentant celle-ci à travers le site de vidéos en ligne YouTube (voir sitographie), à des échanges à son sujet sur le média social Facebook. Afin de retracer les étymologies des phytonymes et des différentes dénominations de la recette, nous avons mobilisé les dictionnaires de différentes variétés de berbère (Dallet 1982 pour le kabyle occidental, El Arifi 2016 pour le berbère de l'Atlas blidéen, etc.), et en particulier les dictionnaires de racines communes (NaïtZerrad 1998, Haddadou 2007).

8 La transcription adoptée dans ce travail, correspond à des alphabets latins adaptés respectivement aux transcriptions phonologiques et scientifiques de l'arabe maghrébin et du berbère-nord. Nous avons également utilisé un système d'abréviation dont voici les différents éléments :

ar. : arabe

$\mathrm{AB}$ : berbère de l'Atlas blidéen

$\mathrm{AJ}$ : arabe jijélien

ber. : berbère

fr. : français

c.p. : communication personnelle

KOC : kabyle occidental

lat. : latin sp. : espèce

TS : tasahlit (ou kabyle extrême-oriental)

Figure 1 : Paysage des Babors, depuis la montagne Agouf (commune de Tamridjet, territoire des Aït Bouyoucef)

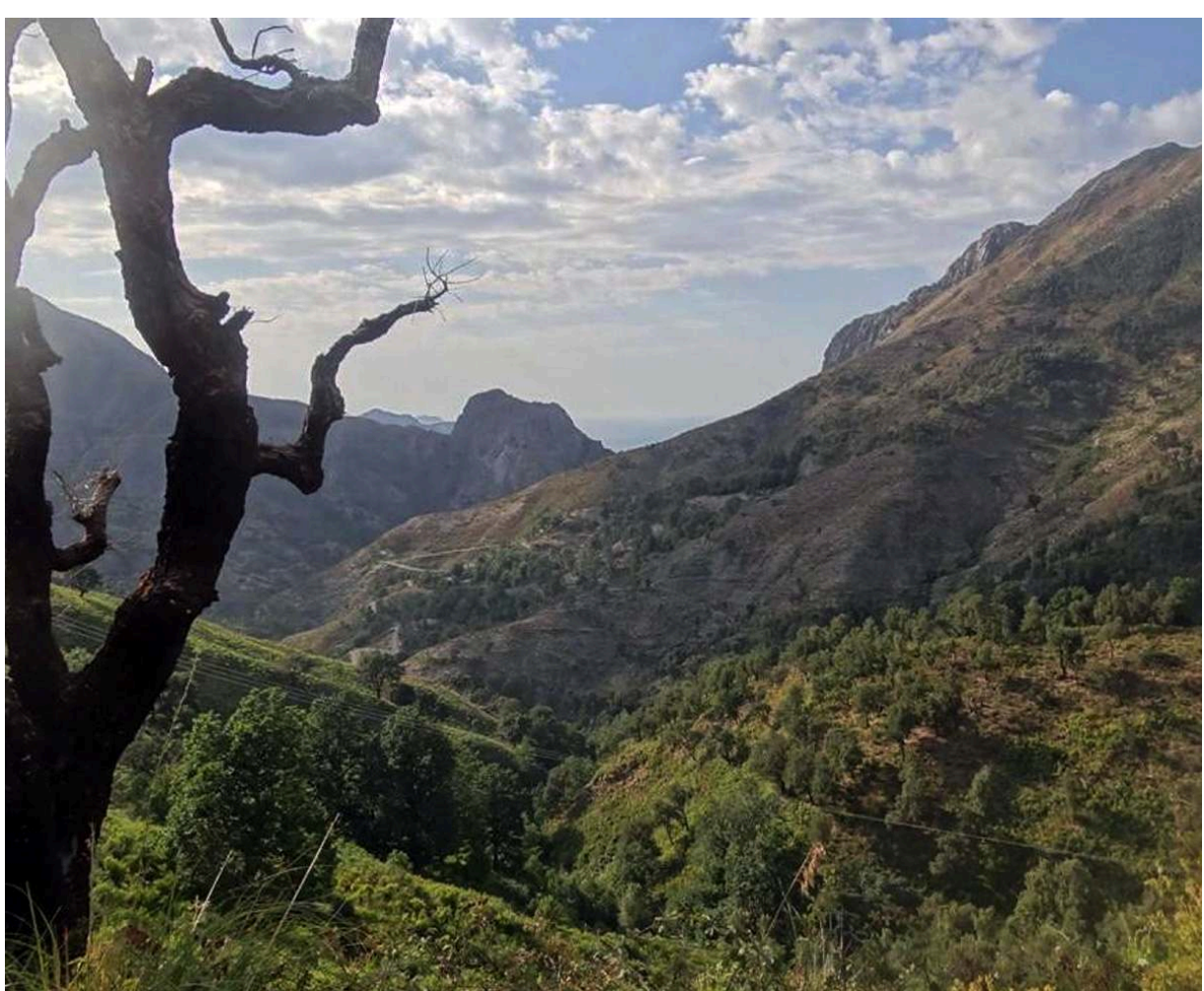

En arrière-plan la mer et les montagnes littorales du territoire des Aït Segoual, les rares habitations se devinent par la seule présence de chemins de bergers et de fils électriques

(c) Garaoun juillet 2017 


\section{La purée d'herbes sauvages des Babors et recettes voisines}

9 La réalisation de purées d'herbes sauvages n'est pas propre au massif des Babors à l'échelle de l'Afrique du Nord. Par ailleurs, d'autres purées végétales, à base d'herbes cette fois cultivées, sont très répandues en Afrique et au Moyen-Orient. À ce titre, les préparations à base de corète potagère (Corchorus olitorius) sont sans doute les plus connues, puisqu'elles sont à l'origine de plats aussi divers que la sauce fakoye au nord du Mali, de la bkaila des communautés juives tunisiennes et plus généralement de la mouloukhiya attestée du Maghreb central au Moyen-Orient en passant par le Tchad et le Soudan.

10 Les équivalents à base d'herbes sauvages sont plus localisés, bien que ceux-ci furent sans doute plus communs dans un passé récent, avant les phénomènes d'exodes ruraux massifs qui ont marqué l'Afrique du Nord dans son ensemble durant le siècle dernier. Notons à ce titre la bakoula marocaine (bəqqūl(a)), véritable plat national, dont l'ingrédient phare est constitué par les diverses espèces de mauves (Malva spp.) que l'on rencontre à l'état sauvage dans le pays. L'avantage certain de l'utilisation de cette plante est son caractère ubiquiste, celle-ci pouvant se développer jusque dans les centres urbains, ainsi que son développement bisannuel permettant de la récolter toute l'année même au cœur de l'hiver. Il arrive que la mauve se suffise comme seul ingrédient de la bakoula, dans ce cas le plat obtenu prend souvent comme dénomination la forme locale utilisée pour désigner ce végétal (à Casablanca xūbbīza désigne la plante et le plat). Dans certaines régions, à la mauve est préférée l'utilisation du pourpier (Portulaca oleracea) : lə-bqūl fih la mauve $w$ ka yəzïdu fih ši bəlea d ər-rbīe ma

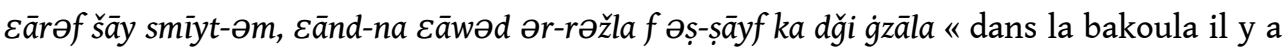
de la mauve et ils ajoutent des herbes dont je ne connais pas le nom, nous avons (y mettons) aussi le pourpier en été, qui la rend très bonne » (A. Hmamouchi c.p., arabe Jbala des Slass). Contrairement à arbiț, les plantes de ce plat sont rarement réduites en purée, le plat est le plus souvent cuit à la vapeur et est agrémenté de nombreux épices et condiments (harissa, citrons confits, cumin, persil, coriandre, etc.). Selon les régions, la bakoula marocaine peut se voir accommoder d'un nombre plus ou moins important de végétaux sauvages, jusqu'à dix-sept espèces d'après l'enquête de Clochey et Aumeeruddy-Thomas (2017) en pays Jbala.

11 En Algérie, plusieurs plats semblables sont attestés à travers les massifs montagneux littoraux. Dans la Kabylie occidentale, la dénomination la plus répandue pour celui-ci est $a^{a b a z i n}{ }^{2}$, le kabyle extrême-occidental lui préfère la forme tagmurt $\underline{t}^{3}$. L'abazin du Djurdjura semble avoir pour ingrédient principal l'ortie dioïque (KOC azegduf, lat. Urtica dioica), présente en grande quantité dans le massif. Selon les régions et les herbes disponibles, on y ajoute quelques herbes sauvages rarement en grand nombre, mais aussi des herbes cultivées fraîches, en particulier de la menthe verte (KOC neEneE, lat. Mentha spicata), du céleri (KOC lekrafez, lat. Apium graveolens), de la bette (KOC tibibidest, lat. Beta vulgaris), des fanes de navet (KOC iferrawen $n$ lleft, lat. Brassica rapa), des gousses d'ail cultivé (KOC tixeddacin $n$ tiskert/ticcert, lat. Allium sativum) et des petits oignons (lebssel d imecțah, lat. Allium cepa). Cette purée est parfois servie comme sauce avec le couscous à gros grains (berkukes), le plat ainsi obtenu est dénommé berkukes weqessul, littéralement, "couscous à gros grain de l'assiette». À l'est du Djurdjura, dans les 
Bibans et la vallée de la Soummam, notre purée végétale porte le plus souvent la dénomination générique de la purée : tahlult ou tahluct. Enfin dans l'Atlas blidéen, une des dernières tribus berbérophones, les Aït Zerman, dénomme becniț ou habecniț un plat de facture très proche auquel on ajoute de l'orge cuit ( $\mathrm{AB}$ mermez, Aït Zerman) et parfois des pommes de terre. Il en existe même une version à base d'écorce d'amande verte dénommée : habecniț n lluz (Samir El Arifi, c.p.).

Dans les massifs des Babors, la purée végétale est réalisée à partir d'un grand nombre d'espèces végétales, parfois plus de vingt, le plus souvent en fin d'hiver ou au début du printemps, lorsque les tiges des herbes sauvages sont encore tendres: taggara $n$ ccetwa

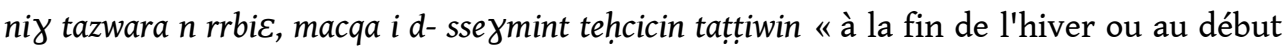
du printemps lorsque les herbes entrent en bourgeon» (TS, Aït Smail). En été et en automne, le plat est réalisé avec les parties restées tendres des mêmes végétaux ou d'une flore à développement estival. À Jijel-ville, dans un milieu urbanisé où la flore sauvage est peu diversifiée, sa confection est élaborée avec des bouquets d'herbes des montagnards : ki nxədmū-h ki ka yəžību-na ən-nās d lə-žbəl əl-ȟš̄yəš d əl-gəmra mən Eānd-həm «nous le faisons (préparons) lorsque les habitants de la montagne nous apportent les herbes du bouquet de chez eux »(AJ, Jijel-ville). Dans certaines régions il peut accompagner un couscous (səksu) fin à base d'orge ou à très gros grains (bərkukəs $b$ arbiț) : on obtient dès lors respectivement səksu bərkukəs b arbiț (AJ).

13 Ce plat est dénommé arbịt à Jijel-ville et dans les tribus environnantes (Bni Qaïd, Oulad Taffer, etc.), tandis que dans l'arrière-pays jijélien et les parlers berbères tasahlit les plus en contact avec l'arabe jijélien (Aït Nabet, Aït Laâlam, etc.), on lui préfère la dénomination hăarbiț. Notons que quelques parlers de l'arrière-pays jijélien appellent ce plat de la même manière que l'on dénomme partout en arabe jijélien le bouquet d'herbes sauvages utilisé pour sa confection : gəmra. À la frontière entre le massif des Babors et celui de Collo, les dénominations ḥărbiṭ et $b q \bar{u} l^{4}$ se croisent et s'emploient indifféremment. Enfin, les parlers berbères zénètes à cheval sur le sud du massif et les hauts plateaux sétifiens (chaoui des Amoucha ou tacemmuct) le dénomment buhellus et les confédérations berbérophones situées à l'ouest des Babors, en contact avec le kabyle de la vallée de la Soummam, tahlult. Devant la diversité des appellations pour ce plat dans les Babors (six formes), nous avons pris le parti d'utiliser dans cet article la forme en usage à Jijel-ville, seule forme à partir de laquelle il est possible de trouver des ressources sur Internet, notamment en termes de recettes.

14 En dehors de sa composante d'herbes sauvages, arbit peut se voir agrémenter de manière tout à fait facultative d'un certain nombre d'ingrédients : parmi ceux-ci, des œufs cuits en omelette (timellalin $n$ tuftayin, TS, Aït Bouyoucef) dans la purée, parfois un morceau de graisse, de la grosse mouture de semoule d'orge ou de blé, plus rarement une poignée de gros couscous ( $\varepsilon i \check{\imath}$, AJ, Bni Mâad). Les seuls condiments attestés parmi les recettes récoltées sont le sel, indispensable, et parfois des piments rouges ou verts (Capsicum sp.). La purée est abondamment arrosée d'huile d'olive de l'année (zzit $n$ tzemmurt $n$ useggas, TS, Aït Bouyoucef) ou de beurre (dhən, AJ, Bni Mâad) pour l'onctuosité ( $t \varepsilon \bar{a} q \bar{i} d a, \mathrm{AJ}, \mathrm{Bni}$ Mâad). On lui ajoute plus rarement du jus de citron (kārrəș, AJ, Bni Hbibi) et la consommation de la purée s'accompagne le plus souvent d'une galette de semoule (tamgadalt $n$ uyrum, TS, Aït Bouyoucef, voir Figures 1 et 2). Enfin, quelques plantes cultivées peuvent agrémenter la composition de arbiț, parmi celles-ci notons l'utilisation courante à Jijel-ville de pommes de terre (Solanum tuberosum) écrasées, celle de féveroles en sauce (abișar) dans la région d'Aokas (Rahmani 1976 : 57), 
mais aussi de gousses d'ail, d'oignons et de feuilles de navet dans l'ensemble de la région, offrant à la composition un goût piquant.

Enfin, la cuisson de arbit peut varier selon l'ordre dans lequel les divers ingrédients sont jetés dans la marmite. Chez les Bni Mâad, on laisse d'abord les oignons et/ou l'ail sauvage jaunir dans l'huile d'olive avant de jeter les herbes qui cuiront dans leur jus, tandis que chez les Aït Bouyoucef les herbes sont jetées dans l'eau frémissante. La variation à ce niveau de la recette pouvant évidemment exister au niveau individuel, chaque famille, chaque cuisinier, ayant ses propres habitudes.

Figure 2 : Purée végétale d'herbes sauvages réalisée par l'auteur de l'article, accompagnée d'une galette de semoule de blé et décorée de fleurs de mauve alcée (TS balaman, lat. Malvea alcea)

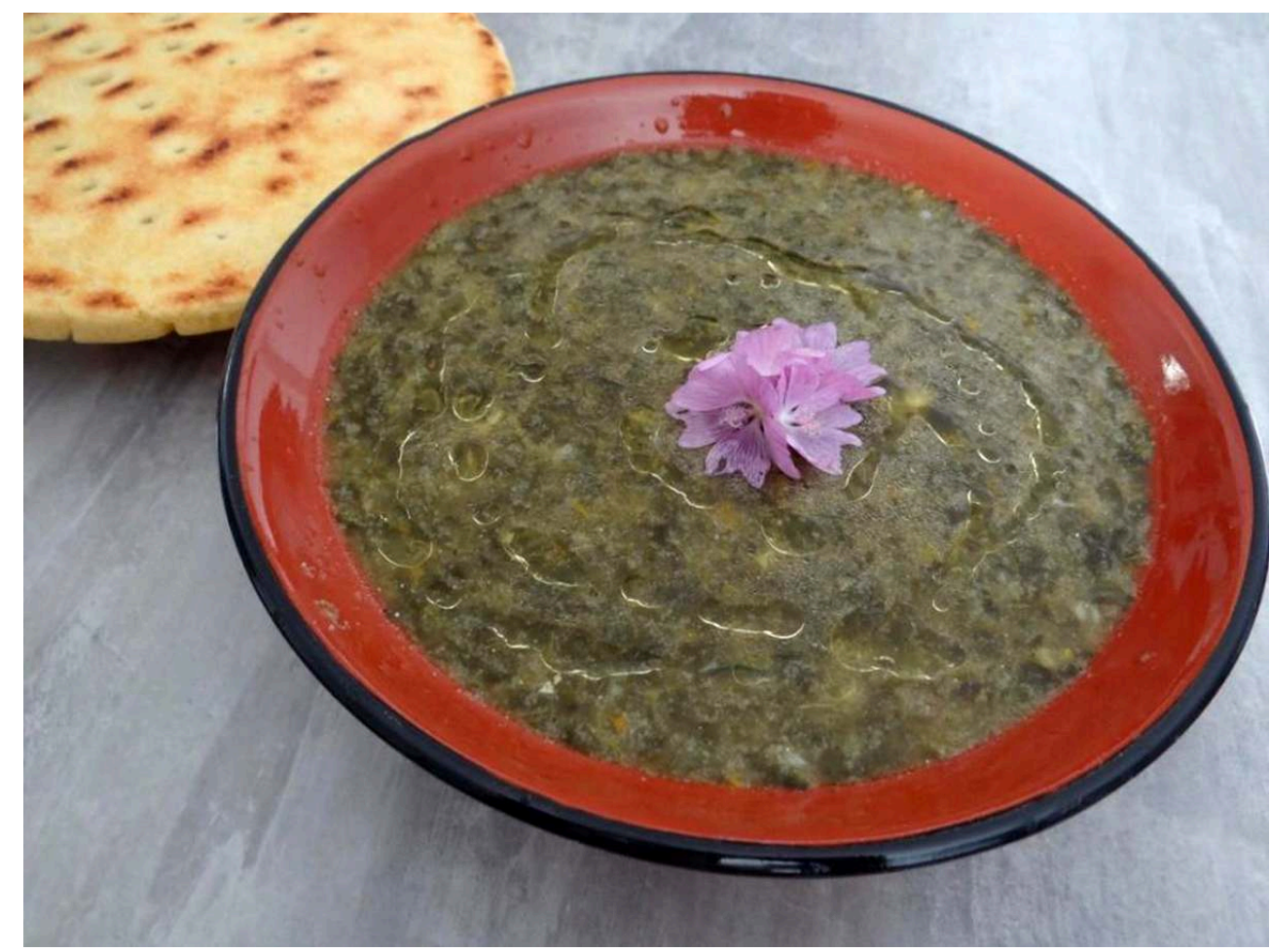

(c) Garaoun septembre 2019 
Figure 3 : Une scène quotidienne de la vie des montagnards des Babors (Aït Bouyoucef, commune de Tamridjet)

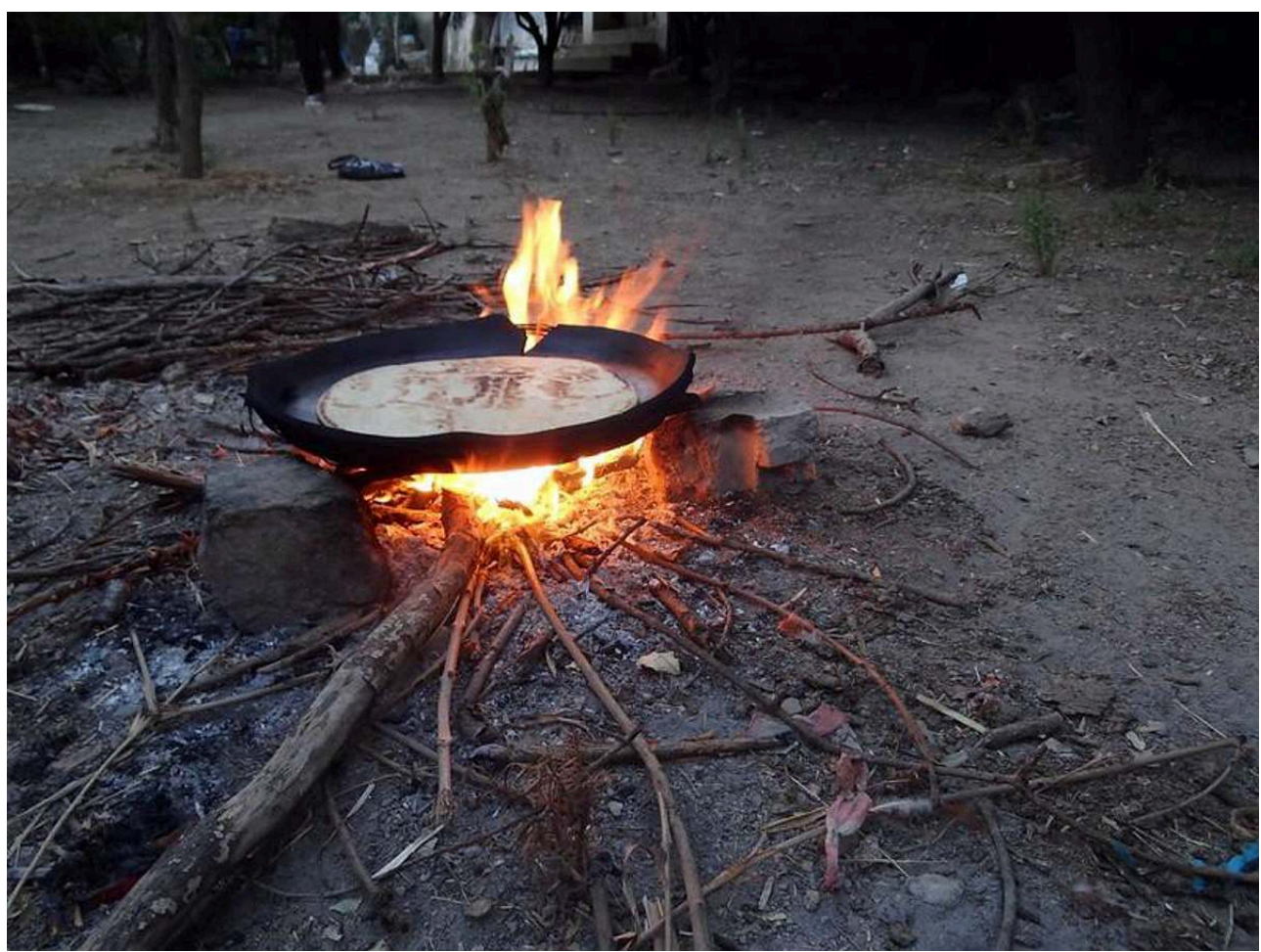

Cuisson d'une galette de pain dur à la semoule de blé (tamgadalt n uyrum iqquren n yirden) sur un feu de bois de foyer domestique (tikerkadiyen n Ikanun), au moyen d'un plat à pain en terre cuite (afan) posé sur trois pierres servant de trépied

(c) Garaoun juillet 2017

\section{Les dénominations de arbiț dans les Babors}

16 Nous discuterons ici les origines étymologiques des différentes dénominations relevées pour désigner les purées végétales à base d'herbes sauvages dans les Babors présentées dans le tableau 1.

Tableau 1 : Dénominations de la purée végétale dans les Babors

\begin{tabular}{|c|c|c|c|c|c|c|}
\hline Dénomination & arbịț & ḥārbīiț & gemra & ahlul & buhellus & bqūl \\
\hline Étymon & $\begin{array}{l}\text { < ber. VBRD } \\
\text { "bouillie " }\end{array}$ & $\begin{array}{l}\text { < ber. VBRD } \\
\text { " bouillie " }\end{array}$ & $\begin{array}{l}\text { < ber. VGMR } \\
\text { " chasser " }\end{array}$ & $\begin{array}{l}\text { < ber. VBLL } \\
\text { " mouiller " }\end{array}$ & $\begin{array}{c}<\text { ber. VBLS } \\
\text { "tas " }\end{array}$ & $\begin{array}{l}<\text { ar. } \sqrt{ } B Q L \\
\text { "gousse" }\end{array}$ \\
\hline Répartition & $\begin{array}{l}\text { Jijel-ville, ses } \\
\text { alentours directs, } \\
\text { et sur le côté est } \\
\text { jusqu'à Ziama }\end{array}$ & $\begin{array}{l}\text { Arrière-pays } \\
\text { jijélien, tasahlit- } \\
\text { Ouest et -Centre }\end{array}$ & Arrière-pays jijélien & $\begin{array}{c}\text { Tasahlit- Centre } \\
\text { et - Est }\end{array}$ & $\begin{array}{l}\text { Chaoui des } \\
\text { Amouchas }\end{array}$ & $\begin{array}{l}\text { Parlers frontaliers } \\
\text { entre l'ar. jijélien et } \\
\text { le colliote }\end{array}$ \\
\hline
\end{tabular}

V(H)RBṬ < VBRḌ (arbịṭ hāābīt) :

Ces dénominations sont les plus répandues dans le massif des Babors et sont l'objet de bien des tentatives d'étymologies populaires, les reliant le plus souvent à des racines de fond arabe de façon plus ou moins fantaisiste: une tendance très courante dans les étymologies populaires de termes arabes maghrébins absents de l'arabe classique. Nous citerons parmi celles-ci la plus sérieuse d'entre elles, reliant ces deux termes à l'arabe VRBT « attacher » (v. Hadji, page personnelle), ce qui aurait été très convaincant si le 
terme désignait le fagot d'herbes sauvages et non le plat cuisiné, la possibilité d'un glissement sémantique n'étant pas à éloigner. La piste d'une étymologie arabe nous semblait probable jusqu'à ce que nos travaux nous amènent à étudier en détail le lexique gastronomique de l'arabe jijélien et à constater la domination écrasante des emprunts directs au berbère : səksu « couscous » (VSKS, Haddadou $2007: 192), b w i \check{s ̌ s} a^{5}$ "plat traditionnel consommé lors de la célébration de l'Achoura, comprenant de la grosse semoule, des dattes et de l'huile d'olive, cuits dans une panse de mouton » (<buar. « celui qui » +-ǐšš « il a mangé » < ber. KC « manger » [TS ecc ečč « manger »] lit. « celui qui a mangé »), zrïr « crème de sésame » (VZR « couler, fondre, boire d'un seul coup ", Haddadou $2007: 239^{7}$ ), rābbūza " purée de navet » (VRBZ « écraser », Haddadou 2007:172), adġəs "plat à base du premier lait d'une vache venant de vêler» (DrS « colostrum », Haddadou 2007:40), etc. Parmi les plats jijéliens dont les dénominations ne sont pas directement empruntées au berbère, on rencontre souvent néanmoins soit une composition comprenant un emprunt à cette langue - $-\varepsilon \overline{a s i i d a ~} d$ ayərni " gouet (Arum sp.) au petit lait » (ayərni agərni $<$ ber. $\left.{ }^{8}\right)$-, soit une des formes "berbérisées » morphologiquement : ağrūf « crêpe épaisse » et axfü « type de beignet », deux vocables de fond arabe (respectivement $\sqrt{ } \mathrm{YRF}$ et $\mathrm{VXFF}$ ) marqués par une voyelle initiale $a$-, préfixe du masculin singulier emprunté au berbère (cf. Garaoun 2018).

19 Arbịt hāarbịt sont les dénominations employées dans la majorité des parlers arabes jijéliens, la première forme, peut-être un peu moins répandue, est utilisée à Jijel-ville dans les tribus des alentours directs (Bni Caïd, Bni Ahmed, Oulad Taffer, etc.) et s'étend à l'est jusqu'aux parlers côtiers de la région de Ziama-Mansouriah (Bni Mâad, Bni Aïssa), tandis que la seconde occupe une large partie de l'arrière-pays : des Bni Hbibi situés sur la rive occidentale de l'oued Kébir, frontière naturelle entre le massif des Babors et celui de Collo, jusqu'aux parlers berbères tasahlit situés à l'est de l'oued Agrioun dans les Babors orientaux (Aït Bouyoucef, Aït Laâlam, etc.). La seconde forme hārbit est souvent considérée à tort comme une déformation de la première : en effet la première syllabe - ha $\bar{a}$ - est, d'après l'étymologie populaire des locuteurs préférant la première forme, le résultat d'une assimilation du numéral un, ḥă, de l'arabe jijélien grammaticalisé comme pronom indéfini devant le nom ārbịt (présenté dès lors comme la forme d'origine). La locution ha ḩārbiṭ « un ārbiṭ » étant même parfois considérée comme caractéristique des parlers de l'arrière-pays. Cette analyse nous a tout de suite paru erronée : une telle assimilation du pronom indéfini ḩā n'étant pas attestée ailleurs dans le lexique de ces parlers, il est difficilement concevable que ceux-ci y aient procédé pour cet unique vocable.

20 La première forme ārbit est quant à elle marquée par le préfixe berbère $a-$, marquant les noms masculin (genre) singulier (nombre) à l'état libre (état) dans cette langue et seulement le masculin (genre) en arabe jijélien. Comme tous les noms jijéliens marqués de ce préfixe, il n'accepte pas l'article arabe. Cet emprunt grammatical de l'arabe jijélien au berbère dont l'usage est attesté à travers un très grand nombre de formes nominales majoritairement de fond berbère, mais également parfois de fond arabe, ainsi «berbérisées", et même d'emprunts à d'autres langues dont des emprunts

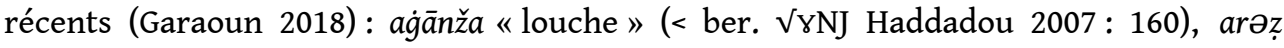
"guêpe " (< ber. VRẒY Haddadou 2007 : 185), afəllūs "poussin » (latin pullus via le berbère), aktūf " épaule d'animal » (vs. ktəf « épaule d'homme » < ar. VKTF), așeyyār «type de tamis » (< ar. ȘYR), atrūtwār « trottoir » (< fr.), apūtu « poteau» (< fr.), ašǐšma « toilette » (< osmanli). 
21 Nous proposons comme origine étymologique du couple arbịt hāärbiṭ la racine panberbère BRḌ, ayant subi d'une part l'assourdissement caractéristique des parlers préhilaliens de la Kabylie orientale ${ }^{9}$, et d'autre part une interversion consonantique : $\mathrm{BRD}>\mathrm{BRT}$ > RBT . La racine BRD renvoie selon les variétés de berbère à ce qui est de consistance semi-liquide, et par extension à toutes sortes de bouillies, purées, mais aussi aux champs sémantiques renvoyant aux selles, flatulences, diarrhées et autres joyeuses expressions du système digestif (Haddadou $2007: 30$ ) : touareg berrit «bruit produit par le fondement, pet ", Moyen-Atlas aserrid «selles liquides », par extension aberdud « queue ${ }^{10}$ et aberriḍ « bouc » chez les Aït Ayyache (Layachi H. c.p.), KOC aberrid «matière fécale liquide, diarrhée ", mozabite) aberḍu « mèche de laine souillée de matière fécale» (Mouh-Mefnoune \& Abdesslam 2011: 21, TS iwerțan urțan «flatulences» ( ${ }^{*} \mathrm{BRD}$ > WRT $\left.{ }^{11}\right)$, aberriț «selle de chèvre ». Cette racine est en l'occurrence attestée en arabe jijélien et en berbère tasahlit à travers le nom à préfixe berbère abərțit bərțiț «boue, gadoue, vase ». Par dérivation verbale, le tasahlit a développé un verbe ssberțeț "embouer, salir de boue", et l'arabe jijélien bərțāt yəbərțəț synonyme. En arabe préhilalien du Rif occidental (pays Jbala, nord du Maroc), on rencontre un autre cognat de cette racine bərțūt « flaque d'eau boueuse ». Le lien sémantique reliant la racine BRD aux dénominations qui nous intéressent pourrait s'articuler à deux niveaux: d'une part à la consistance semi-liquide de notre purée, consistance qui caractérise également la plupart des sémantismes dont dérivent les formes issues de BRD. D'autre part, en raison de l'utilisation de ce plat comme diurétique désintoxiquant, pouvant provoquer la diarrhée, ce qui relirait arbị̣ hāārbịt précisément aux dérivés de BRḌ renvoyant aux selles liquides ${ }^{12}$.

Concernant l'interversion consonantique : BRD > BRṬ > RBT , il convient de noter que les métathèses sont très courantes en berbère :

VMrY « croître » = KOC $m \gamma i$ « germer » / TS $\gamma m i(* \mathrm{MrY}>\sqrt{ } \mathrm{YMY})$

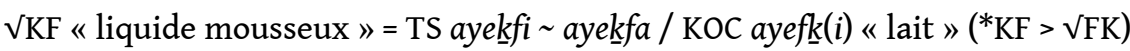

Ce phénomène intervient également auprès des emprunts au berbère de l'arabe maghrébin: AJ (Jijel- ville) būryāmān «inule visqueuse (Dittrichia viscosa)»< bu- ar. «celui qui »+ MGR ber. « rencontrer»+M ber. « eau»; répondant au schéma d'évolution diachronique suivant :

*MGRM (forme en usage en synchronie en TS des Aït Bouyoucef amagraman)

> $b u+$ MGRM (insertion du préfixe arabe bu-; en usage en synchronie en AJ des Bni Mâad : būgrāmān)

$>$ *bu + MYRMN (évolution /g/ > /y/; en usage en synchronie en AJ des Bni Ketit: bāyrāmān)

$>b u+$ RYMN (métathèse)

Enfin, pour ce qui est de la première radicale /ḥ/ de la forme ḥārbīt, signalons que le phonème / $\mathrm{h} /$ est, en berbère-Nord, un emprunt sans doute assez ancien à l'arabe (voir Kossmann 2013 : chap. 5), devenu productif dans la génération de lexique expressif par insertion ou affixation de celui-ci, voir par remplacement de radicales d'origines par celui-ci ${ }^{13}$. Le berbère tasahlit offre un nombre important de termes auprès desquels l'insertion ou l'affixation du phonème d'emprunt $[\mathrm{H}]$ est constatable : tasahlit aguh «brouillard»< ber. VHW «brouillard » ${ }^{14}+/$ h $/$; tasaḥlit ahallum « singe » mais azellum "saignée »: les deux termes dérivant d'une même racine d'origine ZLM (Haddadou 2007 : 234-235) dont la radicale a été remplacée par un /ḥ/ expressif (*ZLM > ḤLM) dans la forme portant le signifiant de « singe ${ }^{15}$. C'est à partir de ces constatations que nous 
proposons comme origine à la radicale $H$ de la variante hărbiț une origine expressive par affixation. Signalons enfin que ce terme n'accepte pas l'article arabe, phénomène caractéristique des termes d'emprunt très anciens en arabe maghrébin: c'est par exemple le cas de l'arabe jijélien bïbba «fait de porter sur le dos » (Jijel-ville dìrl-u ha bibba «porte-le sur le dos»; < ber. VB Haddadou 2007: 23), ou encore de l'arabe marocain $m \bar{u} k a$ « chouette » (< ber. WK, Haddadou $2007: 210$ ).

$\operatorname{VGMR}($ gəmra)

Cette seconde racine ne pose quant à elle aucune difficulté sur le plan de la restitution étymologique. Elle n'est utilisée pour désigner le plat cuisiné que dans quelques parlers de l'arrière-pays jijélien, sans doute par élargissement sémantique. En effet, partout ailleurs en jijélien gəmra $\sim$ yəmra ${ }^{16}$ désigne la botte d'herbes sauvages coupées, prête à être utilisée dans la confection de la purée végétale : əl gəmra ay d kāməl lə-ḩ̌̄āyəš əddi ka yədiru bī-həm arbiṭ (AJ, Jijel-ville) «la gəmra, c'est la totalité des herbes avec lesquelles on fait arbịt ». Par ailleurs, l'arabe jijélien connaît également un verbe gəmmār / yəgəmmər ( yəmmār / iyəmmər) « couper les herbes sauvages » utilisé essentiellement dans le cadre de la recherche des ingrédients de notre recette. En berbère TS, le même verbe gemmer désigne le fait de cueillir à la faucille (des herbes, des céréales, etc.; nom verbal agmer). Le parler kabyle extrême-occidental des At Amran emploie une forme correspondante - tagmurt - pour dénommer le plat.

Cette racine tout à fait pan-berbère renvoie selon les parlers à la chasse, à la pêche, et/ ou à la récolte des fruits, des légumes et des herbes: Kabyle (Haddadou $2007: 75-76$ ) gmer « cueillir, cueillir des feuilles de cardon, butiner », tanegmart « jeune femme qui ramasse, qui cueille ", Moyen-Atlas gmer « chasser, dérober, voler du gibier ", tagemriwt "proie gibier", chleuh $g^{\circ} \mathrm{mr}$ " chasser", tag ${ }^{\circ} \mathrm{mrt}$ "chasse", angmar "chasseur", Ghadamès eğmer «cueillir (en choisissant) des fruits, des dattes". Notons que la seconde radicale est parfois géminée assez indifféremment selon les parlers : touareg tagmert «chasse » / pl. tigemmar, Ghadamès ağemmer «ancienne fête célébrant la première cueillette des dattes ».

$\sqrt{H} \mathrm{LW}<\sqrt{ } \mathrm{BLW}$ (tahlult)

Cette racine pan-kabyle est employée parmi les parlers tasahlit -Centre et -Est au moyen du nominal ahlul connu dans toute la Kabylie le plus souvent au féminin (tahlult $\sim$ taḥlewlt $\sim$ tahluct), le plus souvent pour le sens générique de «bouillie, purée ». Des formes verbales à relier à celles-ci sont également attestées dans beaucoup de parlers kabyles : TS ḥluli « être trempé, devenir presque liquide, être claire (potage, soupe)», ssehluli «tremper, liquéfier ». Ce type de base consonantique comprenant une radicale empruntée à l'arabe (ici [H] ) correspond en berbère-Nord soit à des emprunts à l'arabe, soit à des racines de fond-berbère dont une radicale d'origine fut remplacée par un phonème emprunté à valeur expressive (voir développement de la racine $V(H) R B T$ < VBRD). En l'occurrence, à notre $\sqrt{ } H \mathrm{LW}$ correspond une racine de fond-berbère BLW dont dérivent des sémantismes identiques : touareg belulu " être très liquide ", sebbelulu « rendre très liquide » (Haddadou $2007: 28$ ).

$\sqrt{ } B H L L S<\sqrt{ } B L S$ (buhellus)

Cette racine est rencontrée dans les seuls parlers de la confédération des Amouchas (ber. IEemmucen), une confédération bilingue arabe-berbère pratiquant une variété de berbère zénète se rapprochant du chaoui, une sorte d'îlot chaouiphone septentrional coupé du reste du bloc chaoui par l'axe Sétif-El Eulma. La forme rencontrée dans ce 
parler correspond encore une fois à un dérivé expressif d'un terme de fond berbère auquel fût infixé un $[\mathrm{H}]$ à valeur expressive (voir développement de la racine $\sqrt{ }(\mathrm{H}) \mathrm{RBT}$ < VBRD). La racine $V B H L L S$ dérive en effet d'une racine pan-berbère VBLS (Haddadou 2007 : 28) : touareg ctebolast «gerbe, botte de céréale ", Ghadamès abelsu "motte de terre enlevée d'un coup de houe », kabyle blules " produire en grande quantité ». Cette racine renvoyant à l'idée de «tas, motte, ramassis de végétaux» est clairement sémantiquement motivée par notre purée d'herbes sauvages. plantes principales. Celles-ci correspondant le plus souvent aux ressources naturelles directement exploitables dans la région en question : à Jijel-ville par exemple, la bette est particulièrement utilisée, sans doute parce que cette plante est cultivée dans les environs directs de la capitale régionale. L'utilisation ou la préférence de certaines plantes ou parties de plante est un phénomène parfois très localisé : chez les Aït Smaïl par exemple, nous avons noté l'utilisation de bourgeons de ronce (tațiwin $n$ ujdar) attestée nulle part ailleurs. D'une manière générale, notre purée s'accommode de quelques espèces principales et d'herbes facultatives selon la saison et des espèces végétales présentes dans les environs. La variation concerne le plus souvent la ou les espèces principales: l'amarante sauvage chez les Bni Hbibi (ablātu ${ }^{17}$, lat. Amaranthus 
blitum), la carline (tigernina, lat. Carlina sp.) chez les Chaouis des Amouchas. Néanmoins, notons que certaines recettes rejettent totalement l'utilisation de telle ou telle plante, ou plus souvent de telle plante sans telle autre. Nous avons à ce titre noté par exemple que les Aït Bouyoucef ne cuisinaient jamais cette purée au moyen de la mauve (Malva sp.), qui n'est guère consommée dans cette confédération. Plus souvent, les règles de cette recette concernent l'association des plantes et de leur saveur, c'est ainsi qu'il est donné ce conseil : lūkān tḩətt səmmūm bla məg்rīs yəži hārṛ w məg்rīs bla səmmūm yəži mārr « si tu mets de l'oseille sauvage sans məğrīs (végétal non identifié), il sera piquant, mais məg்rīs sans l'oseille sauvage et le résultat sera amer ».

Le tableau 2 présente les dénominations parmi les parlers étudiés des treize espèces végétales qui sont le plus souvent apparues parmi les différentes recettes récoltées :

Tableau 2 : Phytonymes de dix herbes sauvages parmi les plus utilisées dans la confection de la purée à travers cinq localités du massif Babors

\begin{tabular}{|c|c|c|c|c|c|}
\hline & $\begin{array}{l}\text { ahlul des Aitt Smaill } \\
\text { (Tasahlit-Centre) }\end{array}$ & $\begin{array}{l}\text { harbiț des Ait Bouyoucef } \\
\text { (Tasahlit-centre) }\end{array}$ & $\begin{array}{l}\text { arbīt des Bni Aïssa } \\
\text { (AJ-Ouest) }\end{array}$ & $\begin{array}{l}\text { arbīț de Jijel- ville } \\
\text { (AJJ-Centre) }\end{array}$ & $\begin{array}{l}\text { harbit bqũl des Bni Hbibi } \\
\text { (AJ-Est) }\end{array}$ \\
\hline Ail triquètre & bibras & bibras & (bībrās) & bībrās & bībrās \\
\hline Coquelicot & hraruc & (qbabuc) & qbābūš & bənnūยmān & $\mathrm{C}_{\mathrm{N}}$ \\
\hline Chrysanthème des champs & bayezdud & bayezdud & būyəzūz & $\rightarrow$ & būzdūd \\
\hline Épervière & $S_{1}$ & taz̧udla & taz̧udāl & ḩrīša & ḥrīša \\
\hline Lavande & leḥlahel & leḥlahel & ləḥlāḥ & ləḥlāḥ & ləḥ̂lāḥ \\
\hline Mauve & mejjir & mejjir & məğğīr & məžžīr & $\longrightarrow$ \\
\hline Moutarde sauvage & acnaf & acnaf & būšnāf & būšnāf & $S_{3}$ \\
\hline Ortie & tizegțeft & azegtuf & azəqțūf & azəkțūf & azakțūf \\
\hline Oseille sauvage & tasemmumt & tasemmumt & səmmūm & səmmūm & səmmūm \\
\hline Sainfoin d'Espagne & tasulya & tasulya & sella & sella & sella \\
\hline
\end{tabular}

\section{L'ail à trois angles (Allium triquetrum)}

Cette espèce d'ail sauvage est très utilisée dans la cuisine kabyle, notamment à travers le plat ayrum l lehwal (KOC) "galette aux ingrédients », un pain dur de semoule fourré des tiges nouvelles de cet ail cueillies avant sa floraison. L'élaboration de la purée végétale à partir de cet ail se fait le plus souvent au moyen des tiges, mais les hampes florales et les tubercules sont également parfois utilisés.

La dénomination de l'ail triquètre ou ail à trois angles est identique dans l'ensemble des parlers arabes et berbères des Kabylie, de l'Atlas blidéen au massif de Collo : bïbrās (AJ). En arabe Jbala, un cognat désigne une espèce d'oignon sauvage bəybrūz bəybrūṣ (Colin 1926 : 57) rapproché du latin bulbus (Ibid. $\left.{ }^{18}\right)$. Nous proposons quant à nous la possibilité d'un étymon berbère BRZ/BRS renvoyant au fait de trier et par extension de défricher (Haddadou 2007: 31): Touareg ebres «trier, séparer ce qui est bon de ce qui est mauvais », ebrez "défricher, couper les arbres», enebraz "défricheur», Kabyle ebrez "séparer, défricher, couper le feuillage des arbres », etc. Cette racine a la particularité de présenter comme radicale finale les deux sifflantes /s/ et /z/, ce qui est également le cas des formes présentées comme des dénominations de l'ail sauvage. Par ailleurs, cette suite consonantique pourrait être à relier à la racine FRS (Haddadou $2007: 61$ ) « couper, tailler, et par extension moissonner, émonder ", à l'origine d'un phytonyme dont on consomme la partie souterraine : chleuh afras « navet blanc».

Notons deux caractéristiques originales présentées par la forme nominale bibras : d'une part le doublement de la première radicale $B$, d'autre part le fait que celui-ci ne prenne 
jamais d'article, ni en berbère ni en arabe. La première radicale B redoublée pourrait originalement correspondre au préfixe arabe bu- " celui de ", qui semble émerger dans le cognat des Bni Snouss : bubris (Destaing 1914 : 282), et donc à une forme d'origine correspondant littéralement à "celui du défrichage, que l'on défriche». En effet, les ails sauvages sont des plantes adventices à tendance envahissante, que le paysan est souvent amené à supprimer lorsqu'il souhaite cultiver une zone laissée en friche.

\section{Le coquelicot (Papaver rhoeas)} dénommer cette plante une racine pan-berbère DYZ (Haddadou 2007 : 45) : Moyen-Atlas 
$e d z$ "piler, broyer, enfoncer ", azduz "masse », rifain azduz "maillet», etc. Le KOC connaît une forme composée wazdud wakli (litt. «le maillet de l'esclave»?) pour la même espèce. L'ensemble des dénominations récoltées dans les Babors correspond donc à une racine DYZ «piler et par extension masse, maillet " préfixée de l'arabe bu"celui de ", sans doute motivées par la forme de l'inflorescence de ce végétal après floraison pouvant rappeler celle d'un maillet.

\section{Les épervières (Hieracium spp.)}

Pour cette petite astéracée, les parlers arabes jijélien et le berbère tasahlit étudiés emploient des racines différentes: de fond arabe en AJ - hrriša < VḤRC «rugosité ", motivée par le caractère râpeux du feuillage d'un certain nombre d'espèces de Hieracium, tandis que nos parlers tasahlit préfèrent une base ZDL que nous soupçonnons être une évolution d'une racine ZẒL observable dans le cognat des Aït Mhend (tazuzla « épervière » Berkaï $2014: 1447$ ), qui pourrait découler de la racine panberbère ZL « être droit » (Haddadou 2007 : 233) : touareg ezzel « rendre droit, redresser une chose », amezzul « homme qui rend droit », azel «branche». Signalons une forme $z u ̄ d ə l$ attestée dans certains parlers AJ.

\section{Les mauves (Malva spp.)}

La dénomination de la mauve est remarquablement stable parmi les parlers observés. La seule variation concerne la seconde radicale géminée /jj/, réalisée ou non affriquée selon les parlers. Notons l'absence d'article auprès de ce nom dans les différentes variétés de berbère comme d'arabe de la région.

La racine à l'origine de cette dénomination de la mauve BJR, est bien connue en berbère-Nord (Naït-Zerrad 1998 : 45) : Aït Menacer amejjir (Destaing $1914: 215)$, chleuh (w)ab(j)jir (w)am(j)jir « mauve », KOC mejjir cuffmejjir « mauve ", abajjer " griffe, serre, grand pied, grosse patte », TS aqejjar "patte de volaille » (préfixe expressif $q$-). Selon nous, cette racine BJR pourrait être dérivée par préfixation d'une radicale expressive $\mathrm{B}^{20}$ de la racine pan-berbère ${ }^{*} \mathrm{SKR}>\mathrm{CKR}$ - CCR (Naït-Zerrad 2000: 297) renvoyant initialement à l'idée de "pointue », et par extension aux «ongles - serres - griffes > pied, patte». Les derniers sèmes de «pied, patte» ont sans doute motivé sémantiquement le passage à la dénomination de la mauve, dont les grandes feuilles arrondies rappellent des pattes d'animaux.

49 *SKR (MC isker « griffe ») > CCR (TS tuccar « serres ») > BCR (MC abaccer « grosse patte ») $>$ BJR (KOC abajjer « grand pied») > MJR (Chleuh amejjir « mauve » Ibid.)

\section{La moutarde des champs (Sinapis arvensis)}

L'ensemble des parlers des Babors emploient pour cette brassicacée une racine CNF. Les parlers arabes Jbalas ont quant à eux une forme așnāb rapprochée du latin: Sinapis «moutarde » (Colin 1926: 71); d'où proviendraient également les formes en CNF avec un traitement phonétique différent (Ibid.). Les parlers arabes jijéliens et colliotes préfixent à cette racine l'élément de fond arabe bu- «celui de ». On nous a également signalé une forme būšnāfən dans un parler arabe de l'arrière-pays jijélien, présentant le suffixe du berbère masculin pluriel -ən (utilisé indifféremment pour le pluriel masculin 
et féminin dans beaucoup de parlers jijéliens). Les parlers kabyles occidentaux mobilisent la même racine à travers la forme wacnaf, et ceux du Chénoua (Dahra) hacennaf(i)t (Laoust 1912 : 137).

\section{L'ortie dioïque (Urtica dioica)}

Les dénominations concernant l'ortie appartiennent toutes à une même racine d'origine KDPF «pincer, pincement» (Haddadou 2007: 92) préfixée du factitif verbal berbère $s(s)$ - sonorisé en $/ z /$ dans les parlers traités ${ }^{21}: 1$ 'ortie correspond donc à travers ces dénomination à "celle qui pince». Cette racine est connue dans l'ensemble des parlers berbères de Kabylie, de l'Atlas blidéen (azeğḍf, El Arifi, c.p.) aux Chaoui des Amouchas (azegtuff) ${ }^{22}$, ainsi qu'en arabe jijélien et colliote. La seconde radicale de notre racine pan-kabyle évolue selon les parlers en [g] par sonorisation, puis en semi-voyelle [y] par spirantisation, et de façon très surprenante en [q] ou en [k] sans doute par réanalyse $d u[g]$ berbère en $[q]$ par l'arabe préhilalien ${ }^{23}$. Ajoutons l'existence d'une seconde dénomination en AJ du nord-est (région de Taher) : məžžūṭ (< Kabyle amejjud/t « galeux » ?24, arabe colliote məžžìța).

\section{L'oseille sauvage (Rumex sp.)}

Végétal dont la dénomination est très homogène dans la région, de même qu'elle l'est généralement en berbère. En arabe jijélien, la seule variation constatée concerne le genre attribué à cette dénomination: traitée au féminin dans le parler d'El Milia səmmūma (Hadji, page personnelle), contre un traitement masculin à Jijel-ville səmmūm. La racine à l'origine de ces dénominations est de fond berbère VSM et renvoie à l'idée d'acidité et d'aigreur et par extension à la jalousie (Haddadou 2007 : 195) : touareg sesmem "être amer", tanesmit "oseille sauvage", chaoui asemmam "aigre, rude", semem "être aigre", smumi "se crisper", tasmumit "jalousie», tasemmumt " oseille sauvage ».

\section{Le sainfoin espagnol (Hedysarum coronarium)}

La racine VSL à l'origine de ses dénominations partout en kabyle (KOC tasulla), en chaoui (des Amouchas tisulla, ailleurs tasulla ; Huyghe 1906:627) et en arabe jijélien ou colliote (solla), appartient au fond lexical pan-berbère. Celle-ci renvoie partout au fait d'être lisse et doux (Haddadou 2007 : 192-193) : touareg eslel « être très lisse et doux au toucher ", taselelet « endroit, surface glissante ", eseli « roche lisse " (Atlas blidéen isli, El Arifi c.p.). Une racine rattachée à cette fabacée en raison du caractère doux de ses feuilles, velues (dessous et bord des feuilles) et lisse (dessus des folioles), qui permettent son identification et surtout son tri face aux nombreuses espèces de fabacées ressemblantes toxiques pour le bétail. Ajoutons que l'AJ et le TS connaissaient tous deux un second vocable tiré de cette base consonantique de fond berbère SL : asalu "chemin tracé dans la neige ». La dénomination berbère de ce végétal a connu une extension remarquable dans différentes langues du pourtour méditerranéen: arabe maltais silla (Souag $2018: 200$ ), italien sulla et espagnol zulla, latin médiéval sylla (Souag 2018 : 200), etc. Ces emprunts au berbère s'expliquent par l'origine géographique de cette plante : l'Afrique du Nord occidentale et la péninsule ibérique. 

raison de leur goût ou de leur aspect moins apprécié peuvent entrer dans la composition de notre purée. Parmi celles-ci, citons la menthe aquatique (Mentha aquatica), assez peu commune dans la région et dénommée au moyen d'une composition de fond berbère dans les parlers pour lesquels nous l'avons relevée : Aït Segoual (TS) amelkessif, AJ tāmglāsif (< VMGR « rencontrer » + VSF « rivière »). Signalons également le poireau d'été (Allium ampeloprasum), dont les feuilles les plus tendres sont parfois utilisées. Celui-ci est dénommé dans tous les parlers pour lesquels nous avons pu récolter son nom à partir d'une racine arabe (كرت) : Aït Mhend (TS) aḱuratt (Berkaï). Notons l'utilisation possible mais peu souhaitable à cause de son goût amer du laiteron maraîcher (TS tiffaf ber.). Chez les Amouchas, la recette s'étend rarement à l'utilisation d'une autre plante que la carline (Carlina sp.), dénommée par ses derniers tigernina, un emprunt au roman d'Espagne. Enfin, une curiosité chez les Bni Hbibi, l'utilisation des jeunes feuilles de tomate (Solanum lycopersicum), partie de la plante pourtant reconnue pour sa toxicité (comprenant de la solanine), dont l'ingestion peut provoquer des nervosités et des maux d'estomac. L'utilisation dans la recette de cette confédération des jeunes feuilles vise à limiter les effets secondaires de sa consommation. comme pour les dénominations gastronomiques, le berbère comme source principale des étymons des phytonymes de plantes sauvages dans le massif, en arabe jijélien comme en berbère tasahlit. Cette domination du berbère, langue autochtone et substratique de l'arabe jijélien, n'est pas très surprenante concernant la flore sauvage. Celle-ci ayant été probablement en partie inconnue des arabophones qui apportèrent l'arabe dans la région, ses dénominations furent directement empruntées à la langue substratique. Néanmoins, en comparaison avec la phytonymie d'autres parlers arabes maghrébins et notamment de parlers préhilaliens connus pour leur degré important d'emprunts au berbère, on constate que le pourcentage d'emprunts au berbère dans ce domaine semble plus élevé ici qu'ailleurs ${ }^{25}$. Notons l'existence de quelques phytonymes de fond arabe ou empruntés à d'autres langues (roman d'Espagne) particulièrement bien répandus dans les parlers de la région et dont la diffusion importante et le faible taux de variation posent question ${ }^{26}$. De même, signalons l'intérêt remarquable des phénomènes originaux et complexes liés au contact de langue telle que la réinterprétation de radicales (ortie), la régularisation d'un schème vocalique pour les différentes racines consonantiques servant à la dénomination d'une même plante (coquelicot), etc.

56 Afin de compléter ce chapitre, voici les listes complètes des herbes servant d'ingrédients traditionnellement utilisées pour la confection de arbịt dans cinq localités :

Aït Bouyoucef (berbère tasahlit, 16 espèces) : azegțuf (Urtica dioica), taxsayt (Cucurbita sp.), lleft (Brassica rapa), tasemmumt (Rumex sp.), bibras (Allium triquetrum), tasulya (Onobrychis viciifolia), tiizmas n temyart (Taraxacum sp.), tazudla (Hieracium sp.), tigernina (Scolymus grandiflorus), acnaf (Sinapis arvensis), fudelgem (Borago officinalis), alhah $n$ umyar (?), sater (Tussilago farfara), lherca (?), tiffaf (Sonchus arvensis).

Aït Smaïl (berbère tasahlit, 13 espèces): balaman (Althaea sp.), fudelqem (Borago officinalis), azerwal (?), talezzaz̧t (Salix viminalis), a yellus umyar (Taraxacum sp.), mekkerfud (Galium album), tizegteft (Urtica dioica), Iles ueerrum (Plantago sp.), bayezdud (Glebionis segetum), hraruc (Papaver rhoeas), ajdar (Rubus sp.), taxsayt (Cucurbita sp.). 
Jijel-ville (arabe jijélien, 13 espèces) : bïbrās (Allium triquetrum), ḩrǐs̆a (Hieracium sp.), məžžir (Malva sp.), būšnāf (Sinapis arvensis), tlūdən (?), məșșāyșa (Plantago sp.), azəkțūu (Urtica dioica), lāblìt (Beta vulgaris), səmmūm (Rumex sp.), səlla (Onobrychis viciifolia), žārniz (Scolymus grandiflorus), krāt (Allium ampeloprasum), tālma (Taraxacum sp.).

Bni Aïssa (arabe jijélien, 10 espèces) : zəqțūf (Urtica dioica), səmmūm (Rumex sp.), žārnīz (Scolymus grandiflorus), qbābūšs (Papaver rhoeas), sāțər ( Tussilago farfara), taẓudāl (Hieracium sp.), məg்rīs (?), žārnīz (Scolymus grandiflorus), məğğìr (Malva sp.).

Settara (arabe colliote, 15 espèces) : səlla (Onobrychis viciifolia) būsəqțāf (Urtica dioica), urdāla (?), būšnāa (Sinapis arvensis), slāq (Spinacia oleracea), ḥlïba (?), tiilfāf (Sonchus arvensis), səmmūma (Rumex sp.), žārniz (Scolymus grandiflorus), hərř̌a (?), xūbbizza (Malva

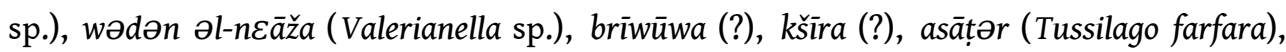
fəržnāṣa (?).

\section{Arbīt dans les traditions et la littérature orale}

L'importance apportée à la purée végétale dans les Babors est mesurable à sa présence dans les traditions, les dictons et les proverbes des parlers de la région. Nous avons à ce titre récolté quelques éléments concernant ces aspects en particulier dans le parler de Jijel-ville, où cette recette est restée particulièrement vivante.

\section{La cueillette}

La cueillette de la gəmra, le bouquet d'herbes sauvages, tend à être abandonnée dans les centres urbains et péri-urbains des Babors, et en particulier à Jijel-ville. Elle y est remplacée par l'achat direct des végétaux auprès de montagnards. Dans les villages, la cueillette des herbes (tawakksa $n$ teymitin, TS, Aït Bouyoucef) se fait encore traditionnellement à la faucille (amger, TS, Aït Bouyoucef) durant tout l'hiver et au début du printemps. Afin de repérer les meilleures herbes et d'éliminer les parties amères ou fibreuses, le cueilleur expert goûte un petit bout de la tige (aġəddu, AJ Bni Hbibi) de la plante en question. On dit également qu'il convient d'attendre une journée ensoleillée pour partir à la chasse aux herbes composant arbiț, une occasion parfaite de se promener en famille et transmettre aux plus jeunes les savoirs botaniques des anciens : dəkān xəržāt əš-šili, əl-gəmra tkūn kbāla «si le soleil est au rendez-vous, la récolte des herbes sera bonne» (AJ, Bni Ahmed).

\section{Les proverbes}

Nous avons également récolté trois proverbes appartenant à la littérature orale de l'arabe jijélien. Les deux premiers sont de Jijel-ville et le troisième de l'arrière-pays :

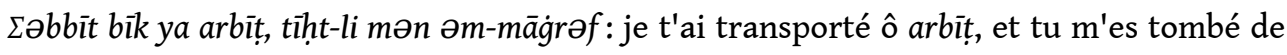
la cuillère.

Xșāāt əz-zəbda farbiṭ : la perte de beurre dans arbịt

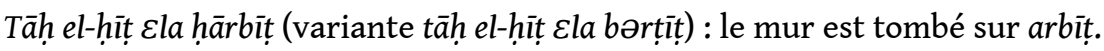

Le premier insiste sur la difficulté de la cueillette des herbes composant la purée végétale, pouvant représenter chez certaines familles jijéliennes une vraie expédition familiale dans l'arrière-pays afin de récolter les précieuses herbes. Le second sur le fait que arbiṭ n'est pas un plat méritant la dépense de beaucoup beurre (une denrée 
précieuse dans la région). Le troisième proverbe contient un sens figuratif qui nous échappe, sa variante employant le cognat bərțịt « boue » n'est pas sans venir confirmer l'association sémantique entre les deux termes apparentés.

\section{Quel avenir et moyens de valorisation}

61 L'avenir de arbiț, comme celui de beaucoup de recettes traditionnelles algériennes rurales, pourrait être menacé à court terme. Dans plusieurs des localités étudiées, nous avons pu constater que les dernières générations ne connaissaient pas ce plat qui, associé à la faim et à la pauvreté, dans des régions où il a longtemps constitué un plat de subsistance et de survie, a aussitôt été abandonné lorsque les conditions de vie se sont améliorées. À Jijel-ville toutefois, la consommation de arbiṭ bénéficie d'un statut de plat qualifié de régional, traditionnel, synonyme de l'éternel lien qui unit la capitale régionale et son arrière-pays montagnard. S'il n'est pas préparé avec autant de ferveur par toutes les familles, arbịt y est sans doute connu par l'écrasante majorité des habitants, au point d'être l'objet de proverbes bien vivants.

Notre travail a mis en évidence la diversité des recettes concernant ce plat, celle-ci variant largement d'un village à l'autre. Dans cette configuration, la disparation d'une variante locale constitue un véritable appauvrissement du patrimoine culinaire de la région, mais aussi des savoirs botaniques liés à cette recette. D'ailleurs, le lecteur remarquera que dans le tableau 2, présentant deux phytonymes des espèces parmi les plus couramment mobilisées dans la confection de arbiț, il existe pour presque toutes les localités citées au moins une espèce manquante, pour laquelle nous n'avons pas su retrouver la dénomination ${ }^{27}$ : indice du lent mais réel affaiblissement de l'expertise des habitants des Babors concernant leurs agroécosystèmes. La transmission des savoirs concernant les environnements naturels des habitants des régions rurales n'étant guère stimulée par le système éducatif algérien, seule une valorisation aux échelles communautaires et familiales a permis la permanence, dans une certaine mesure, de cette transmission jusqu'à nos jours. Il est cependant très incertain que ces recettes perdureront en l'absence totale de statut et leur étiolement, déjà visible, ne pourra être enrayé qu'avec un nouveau regard porté sur les savoir locaux, l'une de nos motivations à l'écriture de cet article.

Nous espérons voir naître dans un futur proche des travaux systématiques sur les différentes recettes de arbiț, ainsi que l'étude de leurs propriétés nutritives. En effet, la consommation de végétaux sauvages, dont plusieurs sont connus pour leurs richesses nutritives, pourrait être considérée comme une forme de régime à part entière ${ }^{28}$, voire d'automédication lorsque la recette contient des espèces végétales reconnues pour leurs vertus médicinales. L'étude approfondie de ces aspects pourrait être particulièrement intéressante en vue d'une valorisation de ce plat et des savoir-faire des montagnards des Babors. Par ailleurs, il serait aussi enrichissant de mieux décrire les différences entre le arbiṭ des Babors et ses équivalents des différents massifs littoraux algériens.

Notons toutefois que arbit, recette probablement de facture très ancienne, s'en sort sans doute assez bien aujourd'hui face aux dizaines de spécialités culinaires des Babors en voie de disparition totale. Ces disparitions touchent tout particulièrement les recettes micro-locales à base de plantes sauvages et d'aventices: lesfenj $n$ ubquq «beignets au gouet» (Aït Bouyoucef), seksu n ubelluṭ « couscous de gland», igisi « lait 
coagulé au moyen de la sève du figuier ", etc. Nous espérons que les montagnards des Babors prendront conscience de la valeur de ce patrimoine exceptionnel et qu'ils sauront enrayer la tendance à l'uniformisation galopante des pratiques alimentaires que l'on observe à ce jour en Algérie.

\section{BIBLIOGRAPHIE}

Berkaï A. 2014 - Essai d'élaboration d'un dictionnaire tasaḥlit (parler d'Aokas)-français. Thèse de doctorat sous la direction du Professeur Md AkliHaddadou, 3 v., Université de Tizi-Ouzou.

Clochey L. \& Aumeeruddy-Thomas Y. - De la façon de nommer aux usages des plantes adventices des cultures en pays Jbala (nord du Maroc). Revue d'ethnoécologie, Supplément 1 : 70 p. [En ligne :] https://journals.openedition.org/ethnoecologie/3065

Colin G. 1926 - Étymologies maghrébines. Hesperis VI : 55-82.

Dallet J-M. 1982 - Dictionnaire kabyle-français: parler des At Manguellat, Algérie. Louvain, Peeters, $1052 \mathrm{p}$.

Destaing E. 1914 - Dictionnaire français-berbère (dialecte des Beni-Snous). Paris, Ernest Leroux, 374 p.

El Arifi S. 2016 - Tamazight de l'Atlas blidéen. Alger, OPU, 768 p.

Garaoun M. 2018 - Noms à préfixes berbères en arabe jijélien. Comparaison entre le parler du centre-ville de Jijel et celui des Bni Mâad de Ziama-Mansouriah, Actes des 20èmes Rencontres Jeunes Chercheurs en Sciences du Langage.

Haddadou M.-A. 2007 - Dictionnaire des racines berbères communes. Alger, Haut-Commissariat à l'Amazighité, 309 p.

Huyghe G. 1906 - Dictionnaire Français-Chaoui. Alger, Adolphe Jourdan, 750 p.

Kossmann M. 2013 - The arabic influence on Northern Berber. Leiden, Brill, 461 p.

Laoust E. 1912 - Étude sur le dialecte berbère du Chenoua comparé avec ceux des Beni-Menaceur et des Beni-Salah. Paris, Ernest Leroux, 228 p.

Mouh-Mefnoune A. \& Abdesslam B. 2011 - Dictionnaire mozabite-français. Alger, ÉNAG, 393 p.

Mourigh K. 2015 - A grammar of Ghomara berber. Leiden, Brill, 544 p.

Naït-Zerrad K. 1998 - Dictionnaire des racines berbères (formes attestées) : A-bezl. Louvain, Peeters, $161 \mathrm{p}$.

Naït-Zerrad K. 2000 - Autours de la base morpho-sémantique SK en berbère Études berbères et chamito-sémitiques, Mélanges offerts à Karl-G. Prasse : 299-301.

Rahmani L. 1976 - Rites et pratiques alimentaires à Aokas (Wilaya de Béjaïa-Algérie). Paris, Université René Descartes, 337 p. 
Souag L. 2018 - Berber etymologies in Maltese. International Journal of Arabic Linguistics 4 (1) :

190-223.

$\underline{\text { Sitographie }}$

Jijel-archeo. [en ligne] Karim Hadji, 2016 [consulté le 10/07/2019]. Disponible sur : https://jijelarcheo.123.fr/repere/index.php?page=arbit

Kabyles de Jijel, Aqbayliyen n Jijel [en ligne] Facebook, 2013 [consulté le 8/07/2019]. Disponible sur : https://www.facebook.com/groups/KabylesCitadins/about/

Lhadra di Jijel [en ligne] Facebook, 2012 [consulté le 8/07/2019]. Disponible sur : https:// www.facebook.com/groups/Lhadra.di.jijel/

رشبة السلق والربكوكس اكلة جزائرية ف الشتاء تفيد مرض الزكام-حاربيط YouTube [en ligne], 2017 [consulté le 11/07/2019]. Disponible sur : https://www.youtube.com/watch? $\mathrm{v}=\mathrm{xN} 4 \mathrm{ZpoKlW80}$

كيفية تحض رب اربيط الاكلة الجيجلية الشه ربة مع نــوارة طبق لذيـذ صح و أقتصـادي [en ligne] Facebook, 2018 [consulté le 11/07/2019]. Disponible sur : https://www.facebook.com/ Jijel.la.merveille18/videos/1806209896067113/?v=1806209896067113

\section{NOTES}

1. L'arabe pratiqué dans le massif des Babors et plus généralement dans la Kabylie orientale appartient en effet aux variétés nées suite à la première vague de conquêtes arabo-musulmanes en Afrique du nord dite préhilalienne ( $\mathrm{VIII}^{\mathrm{e}}$-IX $\mathrm{X}^{\mathrm{e}}$ siècle), tandis que le berbère est la première langue attestée dans le massif depuis l'Antiquité au moins, par les récits épigraphiques romains.

2. Dénomination de fond berbère ( $\sqrt{ } B Z N$ voir Naït-Zerrad $1998: 152)$ renvoyant à différents types de plats selon les régions : chaoui bazin " galette coupée en morceaux trempée dans un bouillon » (Ibid.), ghadamès elbazin "plat de farine mise en boulettes, aplaties et jetées dans de l'eau bouillante et consommées avec de l'huile ». Cette racine est également passée en arabe maltais, tunisien et libyen, où bāzīn(a) désigne la polenta ; chez les arabophones du Rif marocain (Jbala) abāzin désigne un couscous grossier rustique (Hamza Layachi, c.p.).

3. Du berbère GMR (voir chapitre sur VGMR).

4. En arabe colliote, de l'arabe البقل "légume, herbe comestible, gousse », également à l'origine du nom du plat marocain bəqqūla. Bqūl pourrait être la forme dominante parmi les parlers arabes préhilaliens colliotes (non documentés).

5. Notons au moins deux variantes jijéliennes : būgəšša et būyəšša.

6. L'étymologie que nous proposons ici peut paraître surprenante au premier abord, du fait de l'utilisation en composition d'une forme verbale conjuguée berbère. Néanmoins, celle-ci a tout de suite convaincu nos informateurs jijéliens habitués à sa consommation, qui nous ont orienté sur la motivation de celle-ci en raison du caractère très nourrissant de ce plat. Cette allégorie à la dimension très nourrissante d'un plat, nous rappelle la dénomination enfantine d'une recette de la Kabylie orientale (taceșbant, boulettes de semoules parfumées) chez les Ait Bouyoucef: lbumbat $n$ lkerc « les bombes du ventre». Lameen Souag (c.p.) nous a également orienté sur la possibilité d'un lien éventuel entre bwišša et les dénominations du carnaval organisées dans différents ksours sahariens algériens lors de la fête de l'Achoura : Figuig (berbère-Nord) berkaccu, Tabelbala (korandjé) gurg ${ }^{w} \partial y \check{s ̌} a$.

7. Le terme est attesté dans toute l'Algérie orientale et en Tunisie.

8. Cette dénomination est assez commune en berbère: Ghomara ayerni (Mourigh 2015 : 524), tachelhit ayrni (Souag 2018:202), quelques parlers KOC ont acerni (évolution YRN > ERN par 
remplacement expressif de la première radicale /y/ en / $/$ /); et passée dans un certain nombre de parlers arabes : plaines atlantiques marocaines yərni, maltais garni (Ibid.). Il s'agit selon Souag (2018:202) d'un dérivé par métathèse de la racine pan-berbère NYR « front » motivée par la forme de l'inflorescence spécifique aux Aracées (appelée spadice). Des parlers de l'ouest des Babors, notamment celui des Bni Hbibi, ont une autre dénomination pour le gouet, également de fond berbère : kərrīwa > VKR « être rond» (Haddadou 2007:99) motivé par la forme de ses tubercules, Ghadamès akerra « morceau dur ", Zénaga kurer " être rond, circulaire, marcher en rond ».

9. Une évolution phonétique à l'origine controversée attestée à la fois parmi un certain nombre de parlers arabes préhilalien (Jijel, Collo, Ghazawat, Béjaïa, Jbala, Bni Yazgha, Bhalil, etc.) et berbères septentrionaux et orientaux (tasahlit, kabyle oriental-Est, chaoui septentrional, Ghomara, Nefoussa, etc.) : voir à ce sujet Kossmann 2013 : chapitre 5.

10. D'où le phytonyme aberdụ izem « plantain » (litt. « queue de lion »).

11. L'arabe jijélien tərța «flatulence » pourrait également provenir de cette racine.

12. Cette hypothèse nous a été suggérée suite à la présentation de ce plat et de ces dénominations lors de la séance du séminaire de linguistique berbère du 8 novembre 2019 organisée par Amina Mettouchi (Paris).

13. Deux autres phonèmes empruntés par le berbère-Nord à l'arabe sont mobilisés dans la

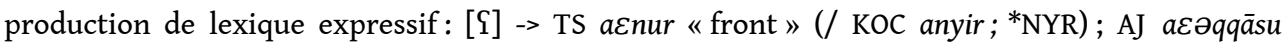
«nourrisson» (*KS; Moyen-Atlas amkkusu, Haddadou 2007:104); et [ $\left.\mathrm{s}^{\mathrm{s}}\right] \rightarrow$ TS aḅalus « vase » (*MLS, TS amillus « escargot »).

14. Voir touareg ahu « fumée » (Haddadou 2007:87), KOC tagut, etc.

15. Notons également parmi les dérivations expressives de cette racine la forme ajehlum « queue » du kabyle extrême-occidental.

16. L'évolution par spirantisation dynamique $\mathrm{du}[\mathrm{g}]$ en [y] est caractéristique des parlers $\mathrm{AJ}$ de la moitié orientale de l'aire de l'AJ, et se constate surtout dans les mots d'emprunt au berbère : Bni Mâad agəns «sol en terre battue de l'habitat traditionnel » / Bni Hbibi ayəns. Cette évolution est également bien connue d'un certain nombre de parlers berbères. En tasahlit, celle-ci touche particulièrement le parler des Aït Ouaret Ouali : Aït Bouyoucef agusim «noix » / Aït Ouaret Ouali ayusim.

17. Signalons cet emprunt remarquable au latin blitum ( $>$ fr. blette) également attesté en kabyle du Djurdjura : blitu (Dallet 1982 : 26).

18. L'évolution phonétique proposée dans ce cas par cet auteur - blbs $>* b b l s>b b r z-$, nous paraît cependant peu probable vue l'homogénéité des formes attestées dans des variétés de berbère et d'arabe très éloignées géographiquement.

19. Suffixe diminutif $\sim$ péjoratif -uc -uk pan-maghrébin.

20. Préfixe décrit par Naït-Zerrad (2000 : 298) comme un augmentatif péjoratif. Il est réalisé / $\mathrm{m} /$ dans les formes du kabyle pour «mauve»; rappelons que $[\mathrm{m}]$ et $[\mathrm{b}]$ sont des phonèmes proches (labiales) très facilement interchangeables en berbère.

21. Nous avons cependant trouvé dans le parler arabe colliote de Settara une forme ayant conservé la sifflante sourde būsəqțāf.

22. D'autres parlers chaouis plus méridionaux préfèrent d'autres dénominations (Huyghe 1906 : 462) : tmeqsin (> ber. QQS « piquer »), timjerredin (> ar. JRD « enlever»).

23. L'arabe jijélien réalise en effet [q] ou [k] selon les parlers l'équivalent de la consonne arabe classique ق. Celle- ci est typiquement réalisée [g] par les parlers arabes dits hilaliens maghrébins. Le [g], très rare parmi les parlers préhilaliens, se rencontre le plus souvent auprès d'emprunts au berbère ou à l'arabe hilalien. En arabe jijélien, une variété d'arabe préhilalien conservatrice du traitement $d u$ en [q] (ou [k]), les emprunts au berbère contenant [g] pourraient voir ce dernier évoluer en $[\mathrm{q}]$ ou $[\mathrm{k}]$ par phénomène de réanalyse, ceux-ci étant traités comme des emprunts à l'arabe hilalien contenant la radicale arabe ق. 
24. Tamazight du Moyen-Atlas amejjuḍ « chauve, galeux » (Layachi H., c.p.), le mot est passé en judéo-arabe marocain sous la forme məžžūt.

25. Voir Clochey \& Aumeeruddy-Thomas (2017) sur les phytonymes des adventices en arabe Jbala (arabe préhilalien des montagnes du Rif marocain), comprenant visiblement peu de termes d'emprunt au berbère: mərriwwa «marrube blanc» (> ber., Marrubium vulgare), azì (> ber., romarin ?), bāgrāmān (> ber., inule visqueuse ?).

26. Doit-on y voir les signes d'une commercialisation par le passé (dénomination homogénéisée par le biais des commerçants)?

27. Et ce même en demandant à des locuteurs âgés.

28. À la manière du régime dit crétois.

\section{RÉSUMÉS}

Une enquête cherchant à relever les différentes recettes d'une purée réalisée à partir d'herbes sauvages dans le massif des Babors en Algérie nous a permis d'étudier sur le plan linguistique différents phénomènes liés au contact entre l'arabe et le berbère dans deux de ses variétés : l'arabe jijélien (žižlìya), et le berbère tasaḥlit (tasaḥlit). En élargissant notre champ d'étude à la description de la place de ce plat dans ces sociétés, et celle de recettes voisines attestées parmi les massifs des Kabylies, ainsi qu'en interrogeant les données linguistiques récoltées et leurs motivations ethnolinguistiques, nous avons été amenés à explorer les champs de l'ethnobiologie et de l'ethnologie culinaire. Nos résultats ont démontré l'importance de la conservation de ce plat pour la transmission des savoirs liés aux agroécosystèmes locaux, dans cette région connaissant un exode rural chaque jour plus important.

A survey looking at the different recipes of a mash made from wild grasses in the Babor Mountains in Algeria allowed us to study different linguistic phenomena related to the contact between Arabic and Berber in two of their varieties: the Jijelian Arabic (əž-Žižlizya) and Berber Tasahlit (Tasahlit). We tried to broaden our field of study to describe that dish - and some comparable recipes attested among the Kabyle mountains -, to describe their places in these societies, and to question the collected linguistic data and their ethnolinguistic motivations, which led us to explore the fields of ethnobiology and culinary ethnology. Our results demonstrated the importance of the conservation of that dish for the transmission of knowledge related to local agroecosystems, in this area, which is experimenting an ever-increasing rural exodus.

\section{INDEX}

Keywords : Recipe, Babor Mountains, Kabylias, Jijel, berber, arab, linguistics, contact, ethnolinguistics, ethnobiology

Mots-clés : Babors, Kabylies, Jijel, berbère, arabe, linguistique, contact, ethnolinguistique, ethnobiologie 
AUTEUR

\section{MASSINISSA GARAOUN}

Doctorant à l'EPHE (LLACAN) en linguistique - 76 La Canebière 13001 Marseille

massinissagaraoun@gmail.fr 